\title{
The Prediction Model of Wind Facility Power Based On ARMA, Neural Network and Wavelet Transform
}

\author{
Feng Lei ${ }^{1}$ and Wang Yuanzhi, ${ }^{2, *}$
}

${ }^{1}$ School of Electrical Engineering and Information Technology, Changchun Institute of Technology, Changchun, 130012, China

${ }^{2}$ School of Computer and Information, Anqing Normal College, Anqing, 246011,China

\begin{abstract}
For the sharp shortage of energy today, it is particularly important making full use of renewable resources-wind energy. An accurate prediction requires being made for the power of wind facility, in order to make the power dispatching department prearrange the wind power dispatching plan according to the change of the power, to ensure the safe operation of power grid and power balance. The establishment of a wind power prediction model cannot be only efficient and reasonable utilization of resources, but also can greatly reduce the adverse effect that wind power fluctuation will brings power balance and frequency on power grid regulation.
\end{abstract}

Keywords: Wind Facility Power, ARMA, Neural Network, Wavelet Transform.

\section{INTRODUCTION}

With the rise of large-scale wind facility, wind energy is increasingly being used to power industry. It also brings a series of problems to the power system, such as voltage, power quality problems and scheduling scheme, especially the unpredictable output power of wind facility brings great difficulty to the operation of power grids. Prediction can not only improve the operation level of the power grid of the wind power output, but also can reduce the consumption of non renewable energy, can improve the economy of power system, and can reduce greenhouse gas emissions. The prediction of wind power starts late in China, not ready fully to work as foreign countries, for example, basic data are not enough for the wind power measuring system needed to prepare, the meteorological information monitoring and the collection techniques are not mature enough for the wind facility, all of these restrict prediction system research and development of wind power. In order to reach the use of wind energy efficient as far as possible, to improve the wind power forecasting accuracy, we have the following problems need to be solved:

The first step: to establish a real-time monitoring of a wind power is given by the model data, here we are using the time series method, the neural network method, the wavelet analysis method, establishing the ARMA model, the model of BP neural network, wavelet analysis model for three different models. On the one hand, the amounts of PA,PB,PC,PD,P4,P58 can be effectively monitored, and can be analyzed its accuracy through comparison with known amounts to calculate the relative prediction errors more than $15 \%$ or not, to test the prediction results meeting the relevant requirements of the prediction precision or not. On the other hand, the highest accuracy model is obtained in contrast to the construction of the three kinds of forecasting models, analyzing their advantages and disadvantages respectively.

The second step: the universal rule is obtained the wind motor group gathering to the prediction of wind power, through comparison of the relative prediction errors of the single wind turbine power(PA,PB,PC,PD)and the relative errors of the forecast of the multi machine total power (P4,P58), analyzing the wind turbines gathering for the prediction errors influence.

The third step: the prediction model of the higher accuracy is obtained further analysis by results. The main factors are analyzed hindering the wind power to further improve the precision of real-time prediction through the established models.

The suitable forecasting model is established making full use of the known conditions. The various nonlinear and discrete should be fully considered in the wind power forecasting, and the given accounts of data not enough should be taken into, because the wind speed has the characteristics of instability, uncertainty, unknown, and there is a great relationship between prediction of the wind speed and the wind power, analysis steps are as follows:

(1) The model is established the approximation change rule of wind power using the known knowledge and contacting the known model;

(2) For pre real-time prediction using the model established, and comparing with the actual values and analyzing the stability; 


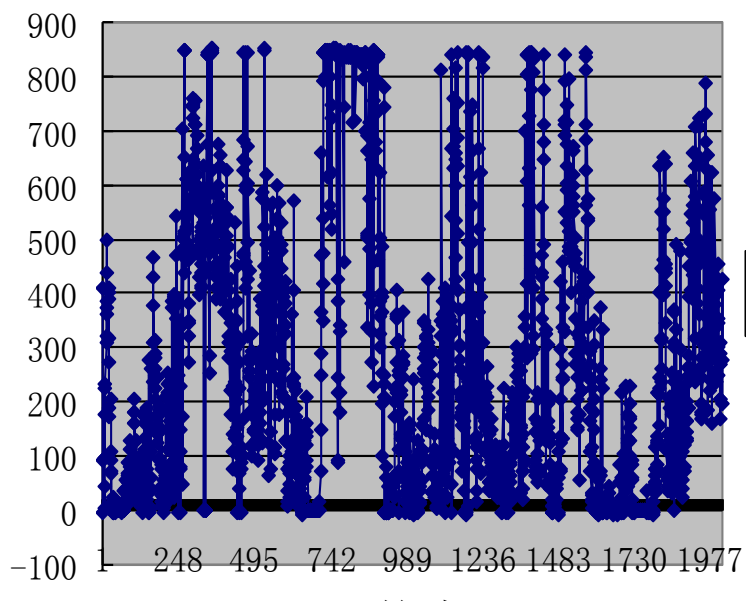

Fig. (1). Data Line Graph of on May 10 to 30.

(3) Build two wind power forecasting models using the same method, on the three prediction models established, compare from various aspects of the errors, the accuracies, the qualified rates;

(4) Analyze the influence of the grid connected power forecasts generated by the bisection through the comparison of the data obtained;

(5) The new model, higher than the three models of Problem One in the accuracy of the proposed, is put forward based on the thought of Problem One because of the important significance of accurate prediction;

(6) Analyzing the main factors hindering the wind power to further improve the real-time forecast accuracy in the process of establishing models.

Process the data in the problem analysis at the same time and feedback the questions of the established models from the resulting data.

\subsection{Symbolic Description}

(1) $t$ is time points per day, of which $0 \leq t \leq 96$

(2) $p$ is autoregressive term in time series model

(3) $\mathrm{q}$ is the moying average term in time series model

(4) $\mathrm{r}$ lis the accuracy rate of predicting plan curve

(5) PMk is the actual average power of the $\mathrm{k}$ period

(6) PPk is the prediction average power of the k period

(7) $\mathrm{N}$ is the evaluation of total numbers of day period (take 96 points -- free assessment points)

(8) Cap is the boot capacity of the wind facility

\section{THE ESTABLISHMENT OF THE ARMA TIME SE- RIES MODEL}

\subsection{Basis of Establishment}

By using time series method, a model can be built for not stationary time series and only need to know a single wind speed or power data of the wind facility simply and good in prediction effect, it has been widely used to predict the wind speed and wind power. Time series model can be divided into autoregressive (AR) model, moving average (MA) model, auto regressive moving average (ARMA) model and the autoregressive integrated moving (ARIMA) model. We choose the ARMA time series model by analysis and comparison.

\subsection{Model Introduction}

The ARMA model is an analysis model proposed by America timing statistician G.E.P.Box and British statistician G.M.Jenkins in twentieth Century seventy's, namely autoregressive moving average model (Autoregressive Moving Average Model), time series prediction method for this model is also called the Box Jenkins (B - J) method. The premise of the ARMA model is that the time series of the established model is generated by a stationary random process with a zero mean. That is, the random properties of the process with the invariance of time show all the sample points randomly fluctuate on a horizontal line in the graphs.

ARMA model is a kind of random time series model in common use, which is currently the most commonly used fitting stationary series model. It can be subdivided into three categories such as AR model (Auto Regression Model), MA model (Moving Average Model) and ARMA model (Auto Regression Moving Average Model).

\subsection{Establishment of Model}

With the PA data as an example, establish the model using the data on May 10, 2006 to May 30, 2006, based on the data of May 31, 2006 as the test data.

Based on the data on May 10, 2006 to May 30, 2006 the line graph is drawn as shown in Fig. (1).

Seen from Fig. (1), fluctuation data is not stable time series data related, therefore the data odd transformation and the differential calculation is needed to carry on. 


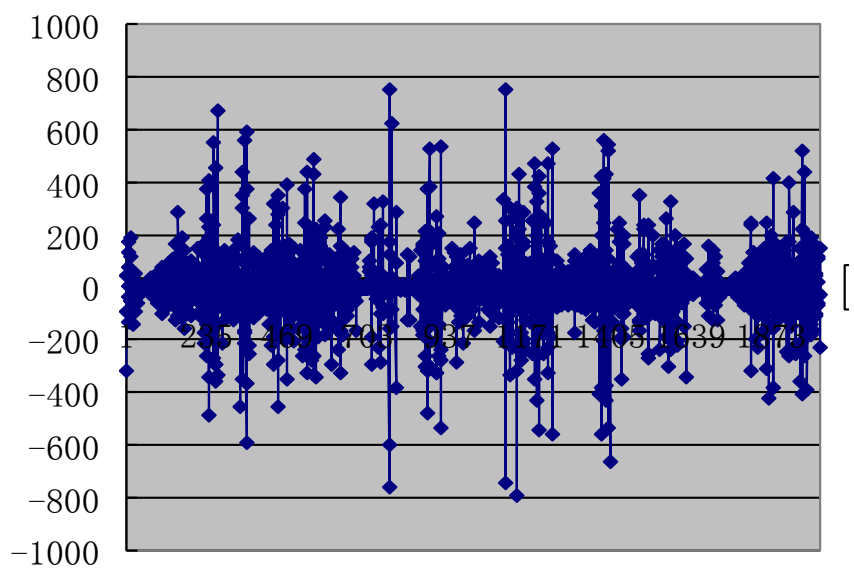

Fig. (2). Data First Order Difference on May 10 to 30.

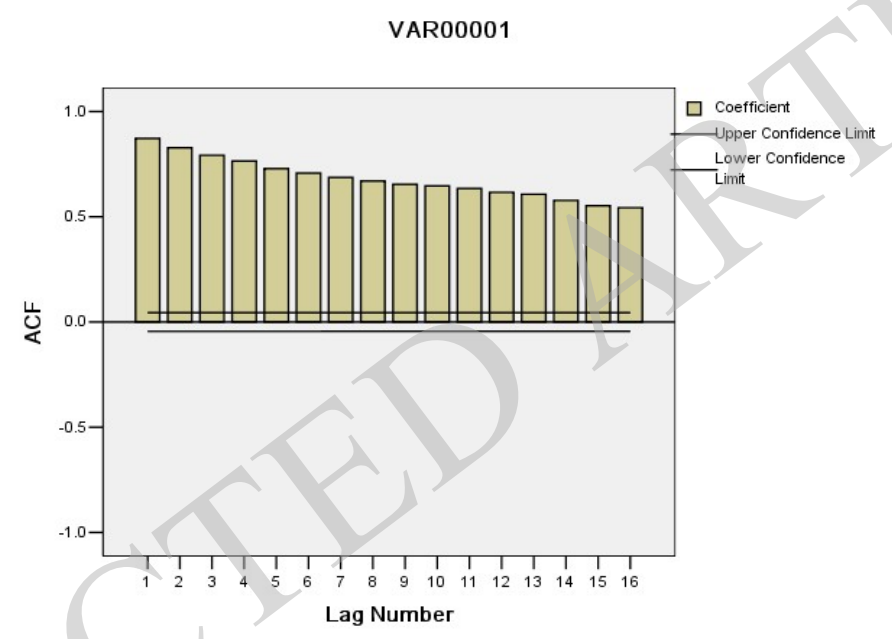

Fig. (3). Autocorrelation Diagram.

The first-order difference of data, the time point data is obtained as shown in Fig. (2).

Seen from Fig. (2), the wind turbine power values always random fluctuations in the vicinity of a constant, and the fluctuation range of bounded, no obvious trend and cycle characteristics, it can be regarded as a stationary sequence and can establish model on the basis of data for further analysis.

We use the SPSS software to draw the correlation diagram further aided recognition; the autocorrelation diagram is shown as in Fig. (3) and Fig. (4).

Seen from Fig. (3) and (4), we can know that the correlation is trailing using the AR (p) model for the autocorrelation function of the stationary series $x(t)$ to analyze data, and the correlation is truncated using the MA model established by partial autocorrelation function, namely AR (p) model is consistent with the time series analysis method, namely establish AR (p) model taking p value 2 .
In the steady model $x_{t}=\varphi_{1} x_{t-1}{ }^{+} \varphi_{2 x_{t-2}}+\ldots \ldots$ $\varphi_{P x t-P}{ }^{+} \varepsilon_{t}$ on either side of the equals sign with rides $x_{t-k}, \forall k \geq 1$, and expectation is:

$E\left(x_{t} x_{t-k}\right)=\phi_{1} E\left(x_{t-1} x_{t-k}\right)+\ldots \ldots+\phi_{p} E\left(x_{t-p} x_{t-k}\right)+E\left(\varepsilon_{t} x_{t-k}\right)$

According to the AR (p) model conditions, $E\left(\varepsilon_{t} x_{t-k}\right)=0, \forall k \geq 1$

So we can obtain the auto covariance function recursive formula

$r_{k}=\phi_{1} r_{k-1}+\phi_{2} x_{k-2}+\ldots \ldots \phi_{P} x_{k-P}$

Due to $\rho_{k}=\frac{r(k)}{r(0)}$ the recursive formula on either side of the equals sign with divided by the variance function $r_{0}$ 
VAR00001

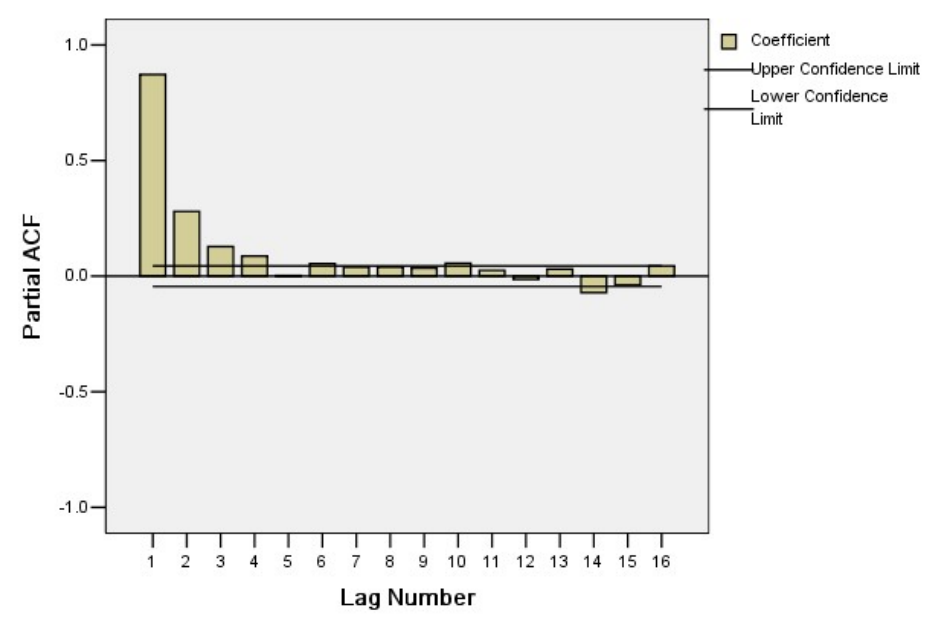

Fig. (4). Partial Correlation Diagram.

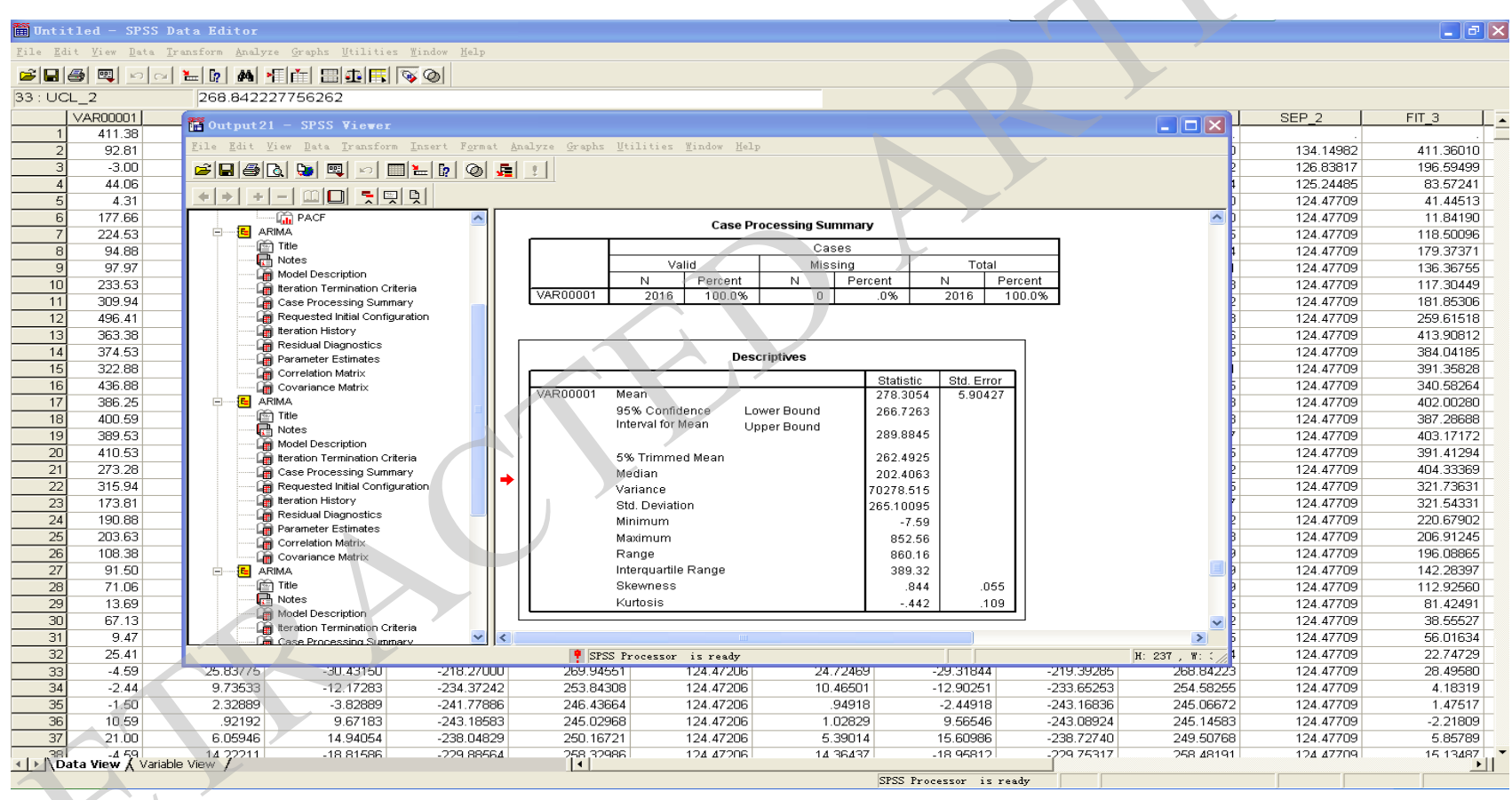

Fig. (5). Results of SPSS Analysis.

auto covariance function, the self recursive formula of the correlation function is obtained

$\rho_{k}=\varphi_{1} \rho_{k-1}{ }^{+} \varphi_{2} \rho_{k-2}+\cdots \cdots \cdot \varphi_{P} \rho_{k-P}$

Based on the above formula can be followed by data calculation, seek $x, r_{k}, \rho_{k}$, then $\underset{\varphi_{1} \varphi_{2}, \ldots \ldots, \varphi_{p}}{ }$ and obtain $r_{1}$, $r_{2}, \rho_{1}, \rho_{2}, \phi_{1}, \phi_{2}$ (5).

By the analysis of SPSS, we obtained as shown in Fig.
From Fig. (5) we obtain the upper bounds and the lower bounds values: 289.8845 and 266.7263 in $95 \%$ the confidence interval.

\subsection{Single Day Power Prediction}

The sequence data forecasts and finishes in 96 time points of PA 0: 0 on May 31st to 23:45 on May 31st as shown in Table $\mathbf{1 .}$

The line graph is as shown in Fig. (6) for the prediction values and the actual numerical values of its 58 turbines: 
Table 1. The sequence data forecasts and finishes in 96 time points of PA 0: 0 on May $31^{\text {st }}$.

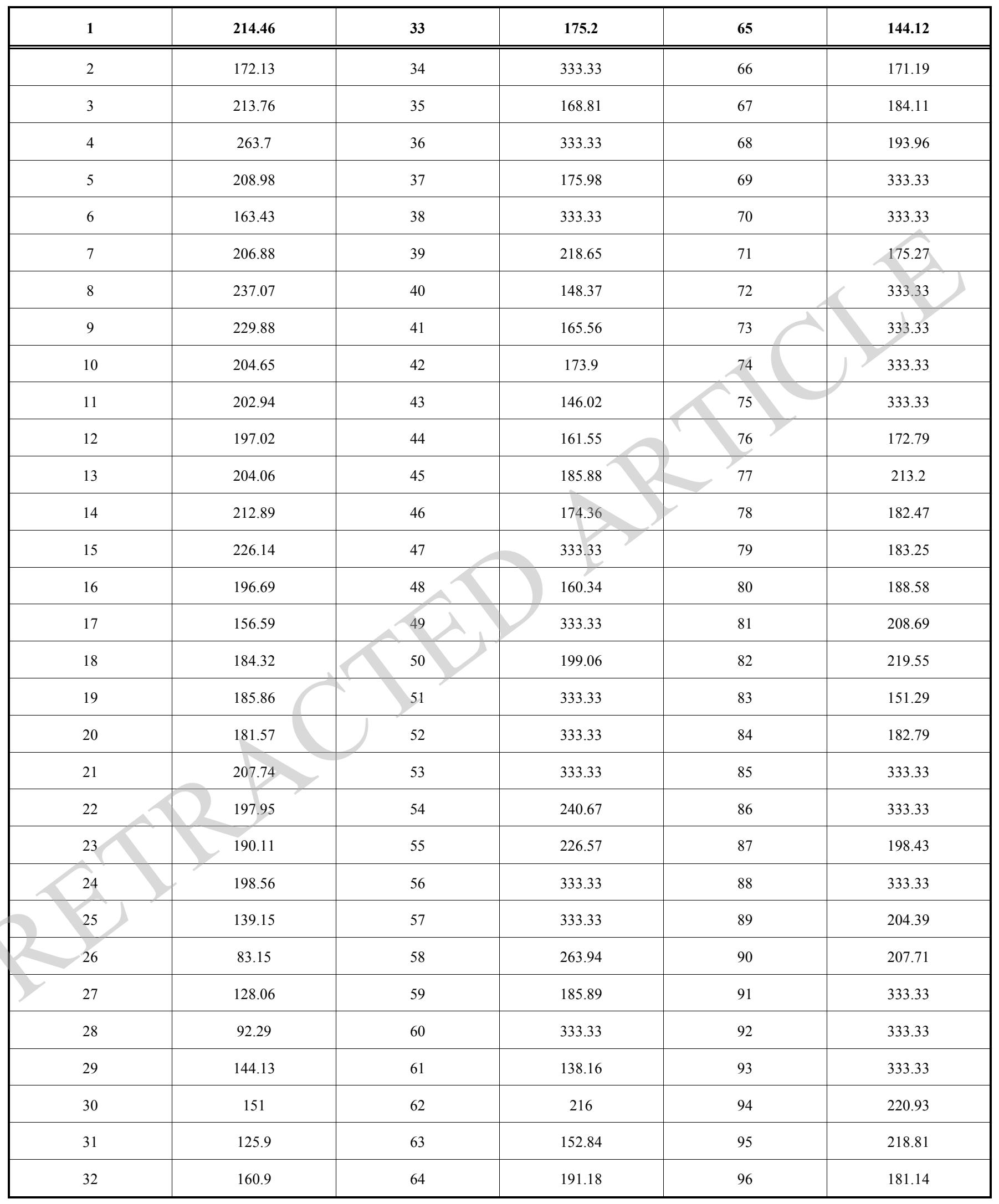




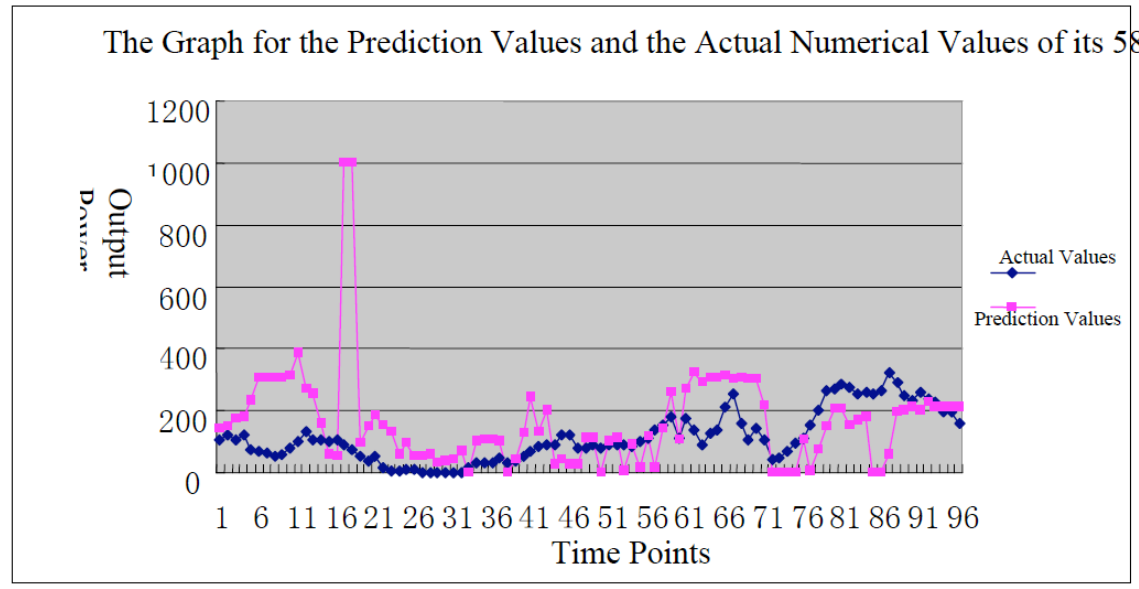

Fig. (6). the Line Graph for the Prediction Values and the Actual Numerical Values of its 58 Turbines.

\section{ESTABLISHMENT OF BP NEURAL NETWORK MODEL}

\subsection{Basis of the Establishment Model}

The neural network model is more suitable for the establishment because the prediction of the wind facility power is intermittent and volatility. The principle of neural networks is to imitate the structure and the function of the human brain, there are a lot of memory, the original connection topology element formed and strong generalization ability, and it is very effective to deal with nonlinear problems. But the chaos on the predictive power of this unknown more wind power very favorable. Among many neural network models, they are more suitable for this case, such as the BP network, RBF network and the selforganizing network. We also compare and select the feed forward error back propagation network called FFBP (Feed Forward Back Propagation) network.

\subsection{Introduction of BP Neural Network}

As a method of wide application range, BP neural network has the characteristics of nonlinear mapping, parallel processing, adaptive learning, robustness and fault tolerance, especially is suitable for the wind power prediction. A typical BP network is three layered feed forward hierarchical network, namely: the input layer, the hidden layer (middle layer) and the output layer. The full connection is carried out between all layers. The sample input signal inputs from the input layer, the hidden layer after, propagate to the output layer, as shown in Fig. (7). In fact, it is to realize the mapping function from the input to the book cart, and the nonlinear mapping complex can be carried out with strong self-learning ability, extension and generalization ability etc.

\subsection{Parameter Setting}

Because the wind power has the characteristics of fluctuation, stochastic and intermittent, the input data type and quantity selected is very important in real time power prediction. This paper takes the wind power 15 days before forecast point as input, respectively predict forecast the predic- tion to 96 time points, i.e. the power data is as input at the same time point 15 days before forecast points, the output power values are as the output by prediction point at this time point. The input layer, the hidden layer and the training function of the output layer, the transfer function, the hidden layer and the output layer node numbers are set as follows:

Training function : trainscg

Adaption learning function: learngdm

Performance function: mse

Layer 2 (input layer): forecast 15 days before $t$ point of wind power

\section{Layer 1 (hidden layer):20 \\ Layer 2 (output layer): 1}

In the neural network setting, we mainly set by the NNTool tool. Matlab7.1 neural network toolbox (Neural Network toolbox) provides an abundant neural network design and an analysis function for the users. Using NNTool in the neural network toolbox to construct a neural network system has the advantages of simple, fast, high precision. From the establishment and the train of the neural network model to the final simulation analysis, the users do not need to write computer programs, the change is simple and easy to modify for the parameters and the models. So, for the setting and the principle of neural network models, we are mainly carried out in Matlab. And in the

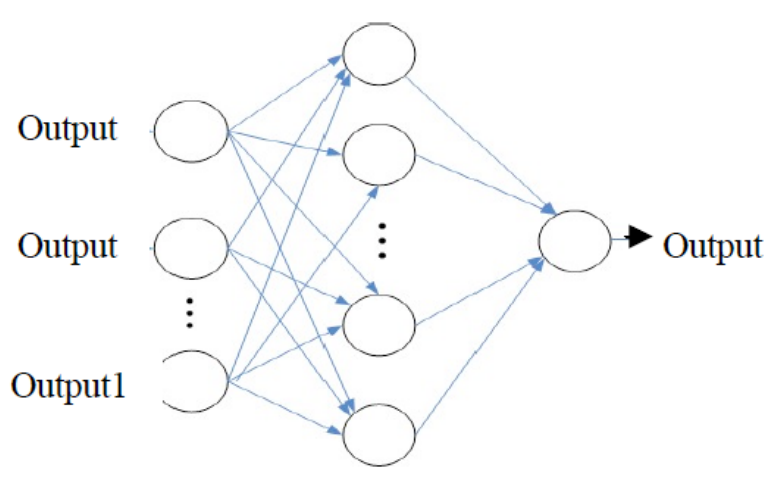

Fig. (7). Schematic Diagram of the Neural Network. 


\begin{tabular}{|c|c|c|c|c|c|c|c|c|c|c|c|c|c|c|c|c|}
\hline Workspace & & & & & $x \times$ & CArray Edite & or - Sheet & & & & & & & & & $\pi$ \\
\hline 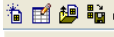 & 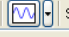 & ck: Base & & & & 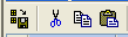 & 回, & $\mid$ t㨽 $^{\text {stack }}$ & Base & & & & & & 円ロ & ㅁ $\square \times x$ \\
\hline Name - & & Value & Class & & & 1 & 2 & 3 & 4 & 5 & 6 & 7 & 8 & 9 & 10 & 11 \\
\hline 田 Sheet1 & & $<28 \times 96$ double $>$ & double & & & 0.47878 & 0.48319 & 0.57703 & 0.47728 & 0.57553 & 0.84394 & 0.84225 & 0.36638 & 0.38669 & 0.62981 & $0.6872 \varepsilon \wedge$ \\
\hline 田 & & $<10 \times 96$ double $>$ & double & & & 0.12628 & 0.24103 & 0.55463 & 0.42975 & 0.32897 & 0.26081 & 0.29691 & 0.24778 & 0.28481 & 0.38081 & $0.5062 E$ \\
\hline 田 2 & & $<10 \times 96$ double $>$ & double & & & 0.12909 & 0.12797 & 0.20681 & 0.23091 & 0.10856 & 0.22069 & 0.11447 & 0.15403 & 0.17325 & 0.23719 & 0.25505 \\
\hline & & $<10 \times 96$ double $>$ & double & & & 0.44344 & 0.35372 & 0.36834 & 0.37894 & 0.49912 & 0.077625 & 0.141 & 0.14944 & 0.19922 & 0.21778 & 0.13584 \\
\hline 田network1 out & & $\langle 2 \times 96$ double $>$ & double & & & 0.17138 & 0.22556 & 0.42169 & 0.27637 & 0.19519 & 0.23128 & 0.21281 & 0.14081 & 0.072844 & 0.049031 & $0.08184 i$ \\
\hline 岗 & & $<1 \times 96$ double> & & & & 0.18609 & 0.27881 & 0.19172 & 0.132 & 0.24666 & 0.20456 & 0.23775 & 0.16519 & 0.25425 & 0.32203 & 0.17981 \\
\hline 田每 & & $\langle 1 \times 96$ double $\rangle$ & double & & & \begin{tabular}{|l|l|}
16 & 0.096844 \\
\end{tabular} & 0.089156 & 0.24197 & 0.33609 & 0.52331 & 0.45497 & 0.30769 & 0.25069 & 0.14888 & 0.11953 & $0.4747 E$ \\
\hline $\mathbb{H}^{\circ} 02$ & & $\langle 1 \times 96$ double $\rangle$ & double & & & 0.39797 & 0.36703 & 0.25622 & 0.23934 & 0.21919 & 0.21 & 0.21666 & 0.23166 & 0.16678 & 0.17597 & $0.2704 \bar{\imath}$ \\
\hline $\mathbb{\boxplus}_{03}$ & & $\langle 1 \times 96$ double $>$ & double & & & 18 & 0.034969 & 0.053625 & 0.042469 & 0.013313 & 0.004875 & 0.016219 & 0.010313 & 0 & 0 & c \\
\hline & & & & & & 19 & 0 & 0 & 0 & 0 & 0 & 0 & 0 & 0 & 0 & c \\
\hline & & & & & & 0.48141 & 0.52519 & 0.44897 & 0.45038 & 0.40022 & 0.34528 & 0.41428 & 0.42056 & 0.5055 & 0.43116 & $0.3917 \varepsilon$ \\
\hline & & & & & & 0.53306 & 0.33591 & 0.47841 & 0.77794 & 0.48216 & 0.19416 & 0.4305 & 0.642 & 0.612 & 0.59878 & $0.4862 \varepsilon$ \\
\hline & & & & & & 0.14119 & 0.38972 & 0.42638 & 0.34163 & 0.56578 & 0.38503 & 0.16612 & 0.43866 & 0.37472 & 0.64969 & 0.70163 \\
\hline & & & & & & 0.27291 & 0.23531 & 0.24206 & 0.28734 & 0.17288 & 0.18188 & 0.16744 & 0.087469 & 0.13931 & 0.19612 & 0.23091 \\
\hline & & & & & & 0.35034 & 0.29559 & 0.29325 & 0.27863 & 0.28153 & 0.30844 & 0.37641 & 0.35813 & 0.43322 & 0.29175 & 0.12431 \\
\hline & & & & & & 0.186 & 0.14006 & 0.025031 & 0.030844 & 0.073781 & 0.098344 & 0.047531 & 0.076313 & 0.11109 & 0.05925 & 0.097031 \\
\hline Current Dire & $-D=\backslash[A T$ & AB7 Y rark & & & $\approx x$ & 0.30956 & 0.32194 & 0.40406 & 0.55828 & 0.43284 & 0.52022 & 0.41522 & 0.31369 & 0.19997 & 0.63891 & 0.60703 \\
\hline (7) $\mathrm{x}$ : & $D=1 \mathrm{MA}$ & SOR & & & & 0.091031 & 0.071063 & 0.055969 & 0.076125 & 0.041906 & 0.036187 & 0.031688 & 0 & 0 & 0 & c \\
\hline (1) 두 & & & & & & 0.27234 & 0.43425 & 0.39366 & 0.46725 & 0.47916 & 0.38409 & 0.46856 & 0.6165 & 0.43059 & 0.50503 & 0.49355 \\
\hline All Files $\triangle$ & File Type & Last Modified & & Description & & 29 & & & & & & & & & & \\
\hline [Hal. als & XLS File & $2011-11-261$ & 00:45 & & 의 & 30 & & & & & & & & & & \\
\hline [to a $2 \times 15$ & XLS File & $2011-11-262$ & 32:07 & & & $<\quad 1$ & ] & & & & & & & & & $>$ \\
\hline a3.xls & XLS File & $2011-11-261$ & 02:10 & & & Sheet1 $\times$ i1 & $\times 12 \times 13$ & $x \quad 01 \times$ & $02 \times 03$ & - & ts $x$ & & & & & 7 \\
\hline [6. a4.xls & XLS File & 2011-11-26 1 & $01: 50$ & & & \begin{tabular}{|l} 
Gommand Iindo \\
\end{tabular} & & & & & & & & & & $\pi x$ \\
\hline [a & $\begin{array}{l}\text { XLS File } \\
\text { XLS File }\end{array}$ & $\begin{array}{l}2011-11-262 \\
2011-11-262\end{array}$ & $\begin{array}{l}52: 05 \\
45 \cdot 30\end{array}$ & & & & & & & & & & & & & \\
\hline i1.mat & MAT-file & $2011-11-262$ & $09: 45$ & & & To get start & ed, select & RATLAB Hel & or Demos & rom the $\mathrm{He}$ & lp menu. & & & & & \\
\hline (192. mat & MAT-file & $2011-11-262$ & $09: 05$ & & & & & & & & & & & & & \\
\hline (19. mat & MAT-file & $2011-11-262$ & $09: 11$ & & & The element tyl & pe "name" m & ust be ter & inated by & he matcl & end-tag & & & & & \\
\hline matlab.mat & MAT-file & $2011-11-262$ & 16:33 & & & Could not parse & e the file: & d: \mat lab & \toolbox \c & slink \o & $k \backslash$ info. & & & & & \\
\hline 01.mat & MAT-file & $2011-11-262$ & 09:18 & & & 》 nntool & & & & & & & & & & \\
\hline (18. 02 mat & MAT-file & $2011-11-262$ & $09: 24$ & & & |〉 nntool & & & & & & & & & & \\
\hline (6. 03 mat & MAT-file & $2011-11-262$ & $09: 32$ & & & $\gg 0$ & & & & & & & & & & \\
\hline (0) pat & MAT-file & $2011-11-261$ & 10:38 & & $v$ & & & & & & & & & & & \\
\hline$\leqslant$ & & & & & $\geqslant$ & & & & & & & & & & & \\
\hline Command History & irectory & & & & & & & & & & & & & & & \\
\hline
\end{tabular}

Fig. (8). Matlab Main Interface.

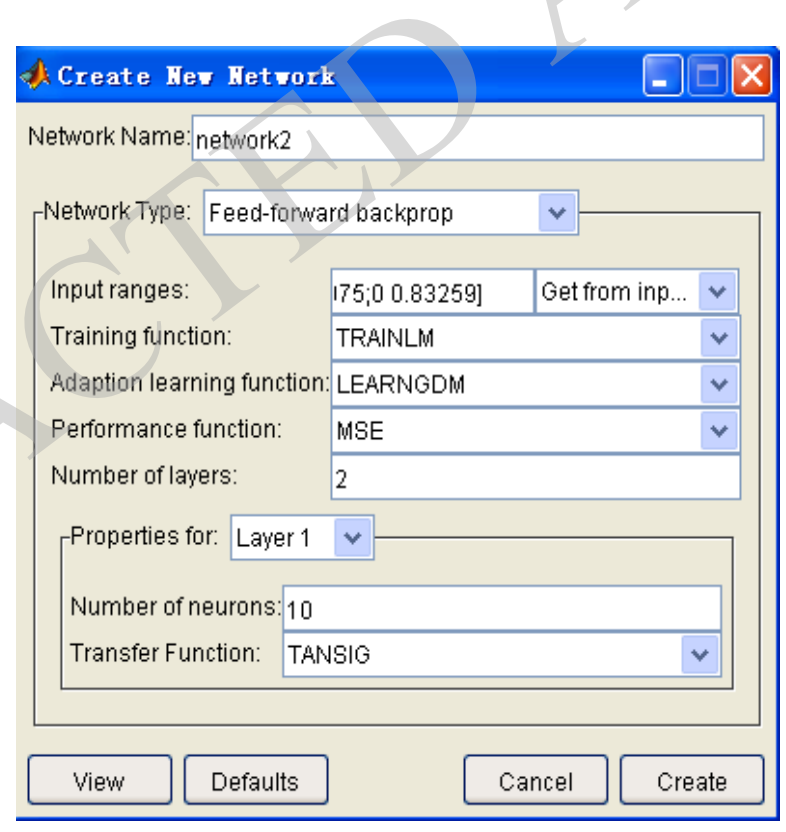

Fig. (9). Neural Networks Creating Interface.

establishment of the network, we use the GUI tools, graphical user interface (Graphical User Interface, also known as the graphical user interface); it is a computer operation user interface displaying using a graphical means.

The Matlab main interface for the establishment of neural network models is as shown in Fig. (8).

Neural network creating interface and neural network interface respectively are as shown in Fig. (9) and Fig. (10).
After the neural network is created, the weights and the domain values of the network have been initialized. The BP network training sometimes needs to reset the weights and the domain in order to avoid falling into a local optimal value. If the weight and the domain values are reedited, then need to be set in Weights options of the neural network structure. The model weights and threshold setting is as shown in Fig. (11). 


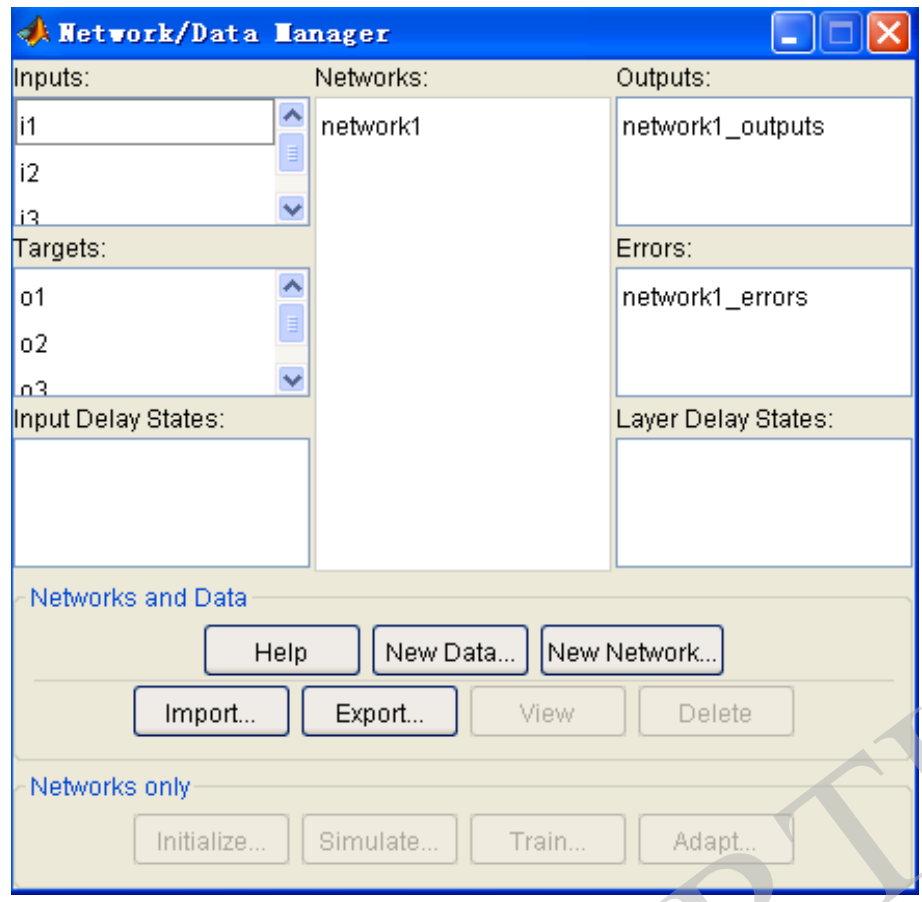

Fig. (10). Neural Network Interface.

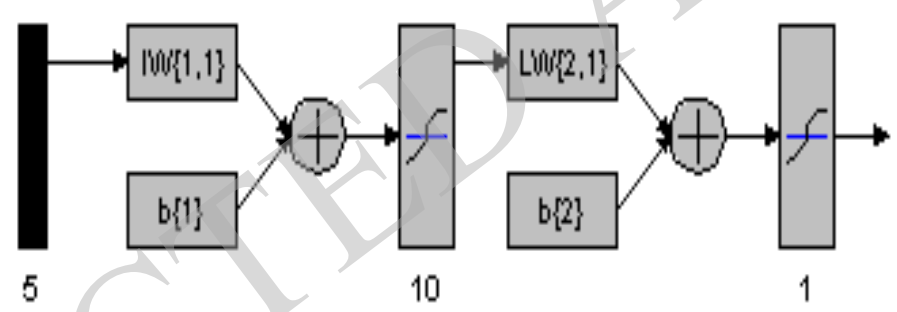

Fig. (11). Weights and Threshold setting.

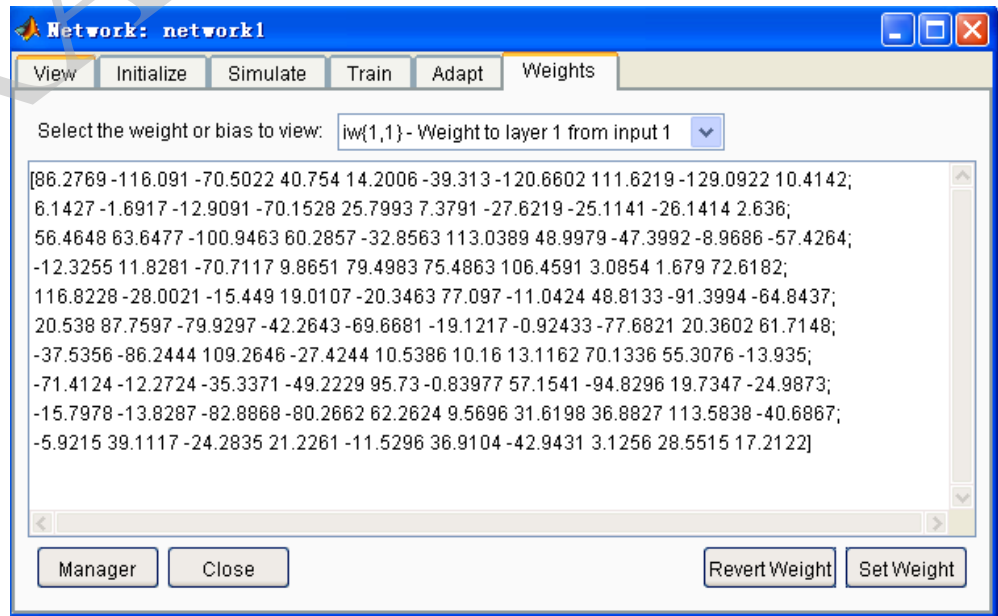

Fig. (12). Right View Interface. 

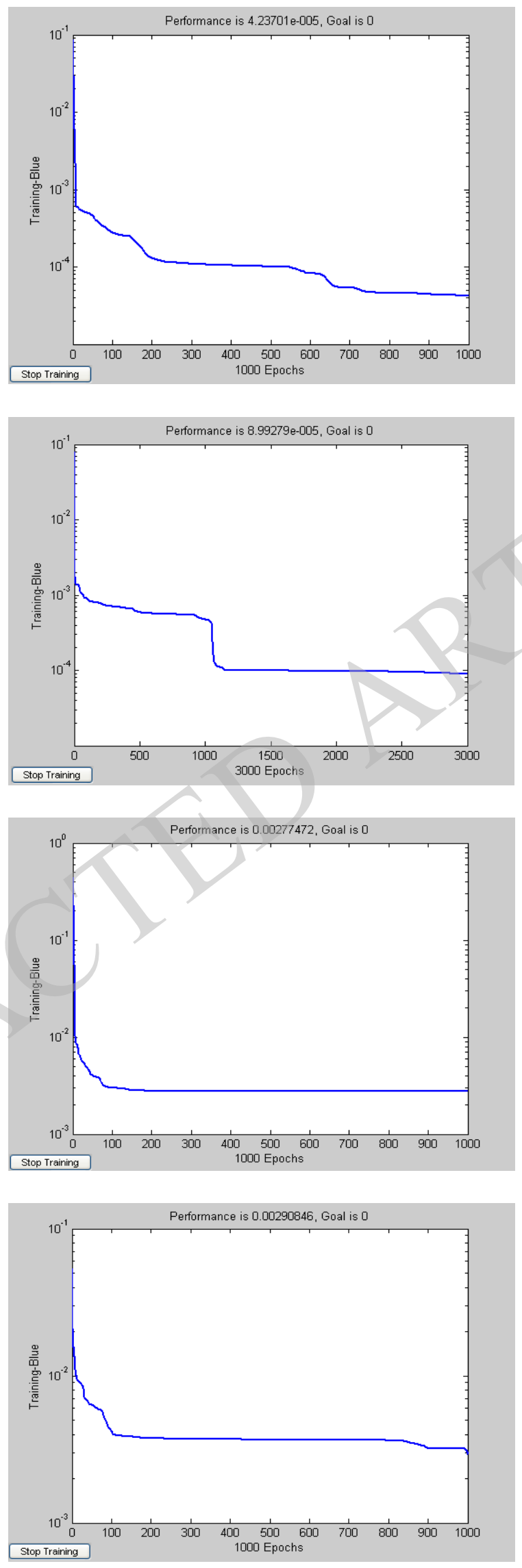

Fig. (13). Training Process Chart. 
Table 2. Total Power Output of 96 Points of 58 Units on May 31.

\begin{tabular}{|c|c|c|c|c|c|c|}
\hline & $\mathbf{1}$ & $\mathbf{2}$ & $\mathbf{3}$ & $\mathbf{4}$ & $\mathbf{5}$ & $\mathbf{6}$ \\
\hline \hline Actual value & 105.84 & 122.98 & 106.97 & 119.56 & 73.577 & 68.075 \\
\hline Predicted value & 143.48 & 149.32 & 174.65 & 179.1 & 232.99 & 305.04 \\
\hline Error value & -37.64 & -26.34 & -67.68 & -59.54 & -159.413 & -236.965 \\
\hline
\end{tabular}

After the weights and threshold are set the right view interface is as shown in Fig. (12).

Main interface you may enter the training page. Set Training Information and Training Parameters, and click Train Network and begin on the network training. At the same time, you can obtain the network training error curve. After several trainings, the training process charts are shown as the following in Fig. (13):

\subsection{Training Process}

After the neural network has been created, click on the

\subsection{Prediction Results and Error Calculation}

Because of the historical power, the wind tower data, the history of numerical weather prediction, wind facility turbine information, operation state and topography will generate the influences for the forecast of the wind power, and we use only the historical power as input in the establishment of prediction model, so the errors will inevitably produce.

After a series of training of the neural network, a trained neural network is given. For example, the input of wind power 15 days before May 31st, the wind power forecast May 31st can be obtained. As the following Table 2:

From the Table 2 data analysis comes to the conclusions that: the neural network prediction results are in the presence of the certain instability, the accuracy rate floating range is larger. The accuracy rate of the maximum is $99.45 \%$; the minimum is only $48.8 \%$.

\subsection{Error Analysis and Improvement Method}

Knowledge based on BP neural network, the analysis for error reasons is: the neural network algorithm is used in the wind power prediction case with a large number of data input, and the training is the decisive causal relationship and the influence between the input data and the output data items desired the input values. And in this model the data input: on the one hand, the amount of data is small, resulting in the insufficient degree of fitting; on the other hand there are not the decisive relationship between data items of the input data and the output expectations values in training. The rate of accuracy is not stable and fluctuation.

In view of the existing problems, put forward the improvement measures for the:

(1) The use of more data input, increasing the sampling days
(2) Using the more data items, such as numerical weather prediction, wind around the terrain information, the unit operation information, and the local climate information and so on.

\section{ESTABLISHMENT OF WAVELET ANALYSIS MODEL}

\subsection{Wavelet Transform Introduction}

Wavelet analysis has the adjustable time-frequency resolution property, the transient characteristics are extremely useful for the analysis of non-stationary signal, but also it has the speed and precision of signal processing obvious aspects. Because of the fluctuation of wind, random, intermittence and the instability of wind power, selecting the approximation of the wavelet analysis model can also be.

Wavelet transform is the local transform of a time (t) and frequency $(w)$, which can effectively extract information from the signal, and the multi scale analysis can be carried on for the function or the signal by translation and dilation. For any function $\mathrm{f}(\mathrm{t})$, the discrete wavelet transforms function:

$$
W T_{f}(m, n)=<f(t), \psi_{m, n}(t)>=\int_{R} f(t) \bullet \overline{\psi_{m, n}(t)} d t
$$

Discrete wavelet function:

$$
\psi_{m, n}(t)=a_{0}^{-\frac{m}{2}} \psi\left(a_{0}^{-m} t-n b_{0}\right)
$$

Discrete wavelet coefficient:

$C_{m, n}=\int_{-\infty}^{+\infty} f(t) \overline{\psi_{m, n}(t)} d t=<f, \psi_{m, n}>\quad$ (take $m, n \in Z^{+}$, assume that $\mathrm{a} 0>1$ )

In 1989 Mallat proposed the concepts of multiresolution analysis, presented to explain the mathematical orthogonal wavelet from the function analysis, obtained the fast algorithm of wavelet transform, the signal $\mathrm{f}(\mathrm{t})$ is orthogonally projected to the two spatial resolutions; according to the resolution $\mathrm{m}$ the discrete approximation signal $A_{m}$ and detail signal $D_{m}$ are obtained. With the increase of $\mathrm{m}$, realize the level decomposition of signal. The decomposition process is as shown in Fig. (14). 


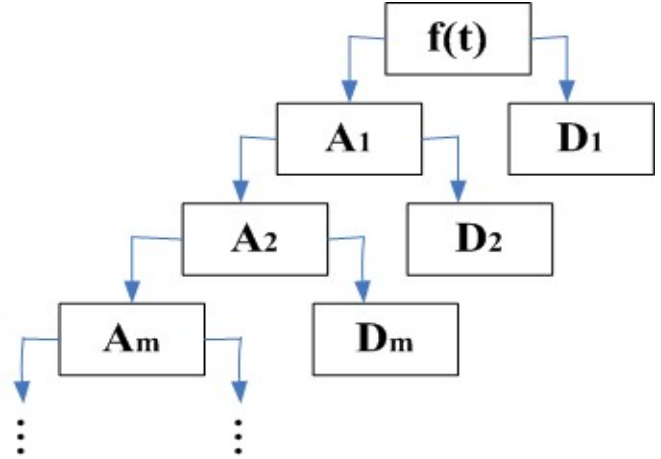

Fig. (14). Schematic Diagram of Wavelet Decomposition Process.

\subsection{Least Square Estimation Theory}

Linear model $y_{i}=\beta_{1} x_{i 1}+\beta_{2} x_{i 2}+\ldots+\beta_{n} x_{i n}+e_{i} \quad(1 \leq i \leq N)$ among them, $y_{1}, y_{2} \ldots y_{N}$ for the observed data, and the independent variables $x_{i 1}, x_{i 2} \ldots x_{i n}$ are known, $\beta_{1}, \beta_{2} \ldots \beta_{n}$ $\beta_{1}, \beta_{2} \ldots \beta_{n}$ are the special parameter estimation, ei is zero mean error not associated, that type can be written as a matrix form:

$$
\left(\begin{array}{c}
y_{1} \\
y_{2} \\
\cdots \\
y_{N}
\end{array}\right)=\left(\begin{array}{cccc}
x_{11} & x_{12} & \ldots & x_{1 n} \\
x_{21} & x_{22} & \ldots & x_{2 n} \\
\cdots & \ldots & & \ldots \\
x_{N 1} & x_{N 2} & \ldots & x_{N n}
\end{array}\right)\left(\begin{array}{c}
\beta_{1} \\
\beta_{2} \\
\cdots \\
\beta_{n}
\end{array}\right)+\left(\begin{array}{c}
e_{1} \\
e_{2} \\
\cdots \\
e_{N}
\end{array}\right)
$$

Or write to $Y=X \beta+e$

\subsection{Wavelet Decomposition Process}

In the inside of the Matlab 96 data of PA on May 10th is carried on two layers of wavelet decomposition, one low-frequency components ca2 and two high frequency components $\mathrm{cd} 1$ and $\mathrm{cd} 2$ are obtained, which are extracted and drawn the low-frequency components and all of highfrequency components waveforms, so as to the reconstructed signal is obtained, and through the process comparing the original signal and the reconstructed signal, the error is finally obtained. The program is established as follows:

96 data of PA on May 10th is carried on two layers of wavelet decomposition, one low-frequency components ca2 and two high frequency components cd1 and cd2 are obtained, which are extracted and drawn the low-frequency components and all of high-frequency components waveforms.

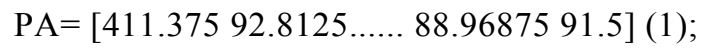

Subplot $(5,1,1)$; plot $(\mathrm{PA}) ; \%$ to draw the original signal waveform PA

Title ('original signal');
[C, L]=wavedec (PA, 2,'db7'); \% of PA by 2 layer wavelet decomposition, if want to change into 3 layers, with 3 providing 2 . Wavelet, $\mathrm{db} 7$ wavelet is used. The decomposition results are preserved in $\mathrm{C}$ and $\mathrm{L}$

$\mathrm{ca} 2=\operatorname{appcoef}(\mathrm{C}, \mathrm{L}, \mathrm{db} 1$ ', 2); \% extract low-frequency component ca2.

Subplot $(5,1,2)$; plot (ca2);

Title ('low frequency component of the ca2');

$\mathrm{cd} 1=\operatorname{detcoef}(\mathrm{C}, \mathrm{L}, 1) ; \%$ extracting high frequency components of $\mathrm{cd} 1$

Subplot $(5,1,3)$; plot (cd1);

Title ('high frequency component cd1');

$\operatorname{cd} 2=\operatorname{detcoef}(\mathrm{C}, \mathrm{L}, 2) ; \%$ extracting high frequency components of cd2

Subplot $(5,1,4)$; plot (cd2);

Title ('high frequency component cd2');

$\mathrm{a}=$ waverec $\left(\mathrm{C}, \mathrm{L}, \mathrm{d}^{\mathrm{d} b 7}\right)$;

Subplot $(5,1,5)$; plot (a);

Title ('signal reconstruction');

$$
\text { Err }=\text { norm }(\mathrm{PA}-\mathrm{a})
$$

Note: (1)for daily statistics after the reconstruction of signals first fit the curves from 96 points for each term. For example, in the Matlab fitting procedure on PA is prepared as follows:

$$
\begin{aligned}
& \mathrm{x}=0: 1: 95 ; \\
& \mathrm{y}=\left[\begin{array}{llll}
483.75 & 399.6563 \ldots \ldots & \ldots 05 & 359.1563
\end{array}\right](2) ; \\
& \mathrm{n}=3 ; \\
& \mathrm{p}=\text { polyfit }(\mathrm{x}, \mathrm{y}, \mathrm{n}) \\
& \mathrm{Xi}=\operatorname{linspace}(0,95,100) ; \\
& \mathrm{z}=\text { polyval }(\mathrm{p}, \mathrm{xi}) ; \% \text { polynomial evaluation } \\
& \text { Plot (x, y, Xi, Z,'k:' } \mathrm{x}, \mathrm{y} \text {, and 'b') } \\
& \text { Legend ('raw data','3 order curve ') }
\end{aligned}
$$

Note: (2)as the daily statistics

The fitting curves and the actual curves of 96 points can be obtained after running the program, and the fitting coefficient. It is shown as the following Fig. (15), respectively the fitting curves of 5 days before $\mathrm{PA}$ and the fitting curves and the fitting coefficients of the first day PB, PC, PD, P58 are as shown in the diagram below:

The fitting coefficients obtained by Matlab fitting are listed in the following Table 3:

In the case of PA, draw the 28 fitting curves of PA as in Fig. (16):

Using the method of least squares fitting into one final fitting curve function is:

$$
y=-0.00087 t^{3}+0.166593 t^{2}-8.66894 t+210.2515
$$


PA1

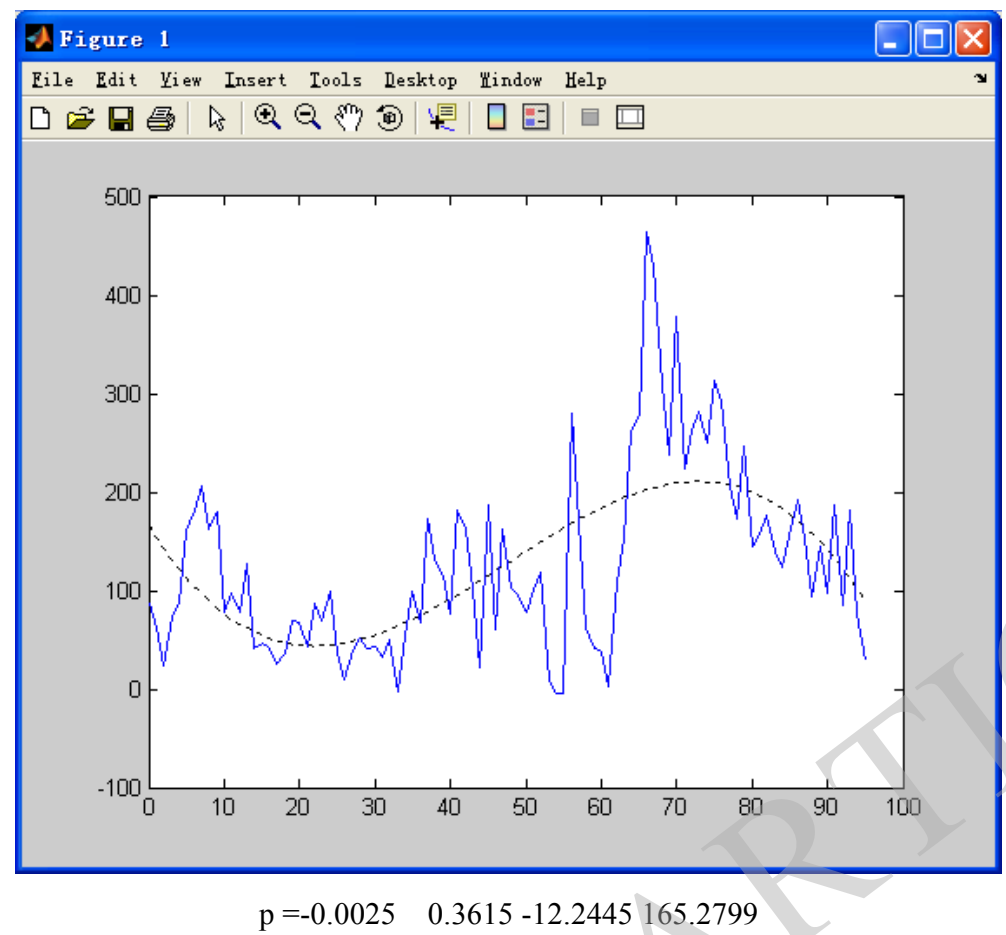

PA2

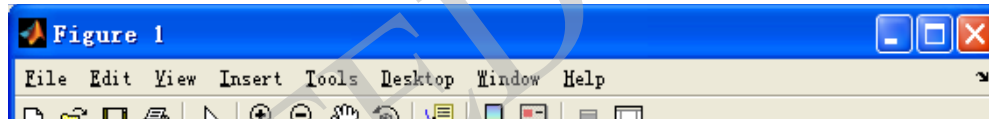

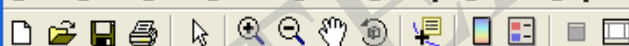

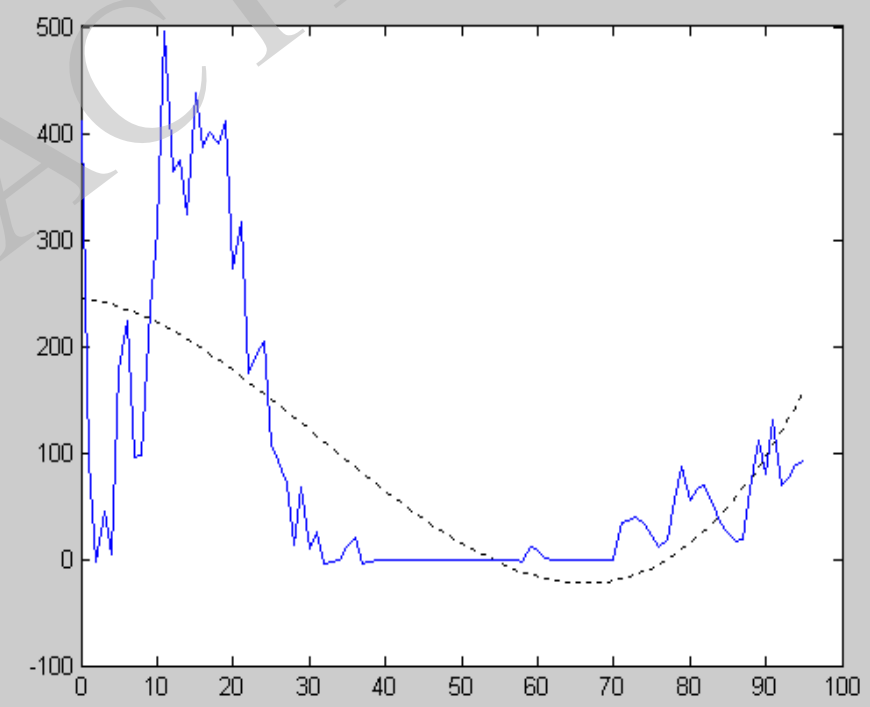

$\mathrm{p}=0.0016 \quad-0.1566 \quad-0.8874244 .6134$

Fig. (15). Contd... 
PA3

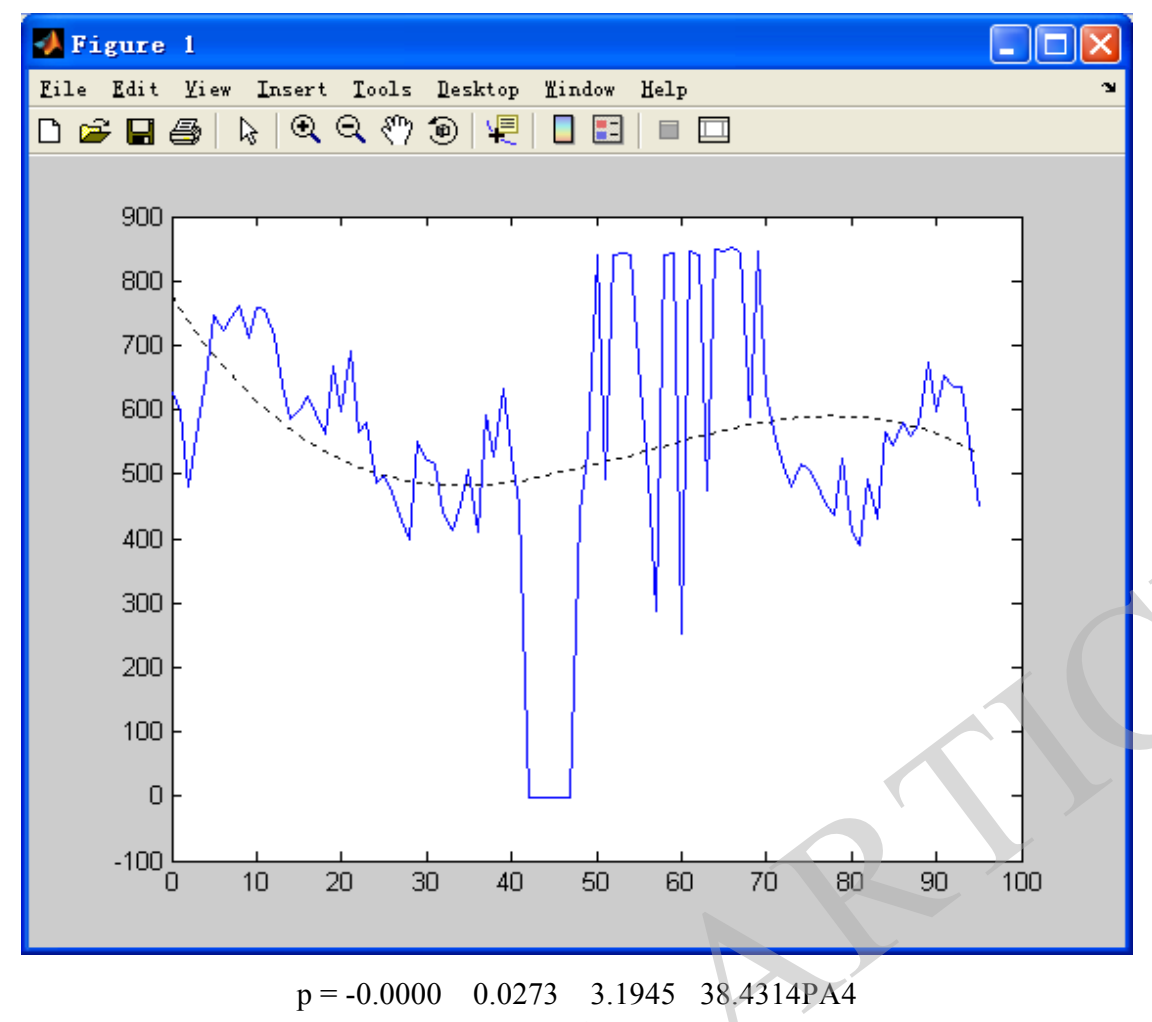

PA4

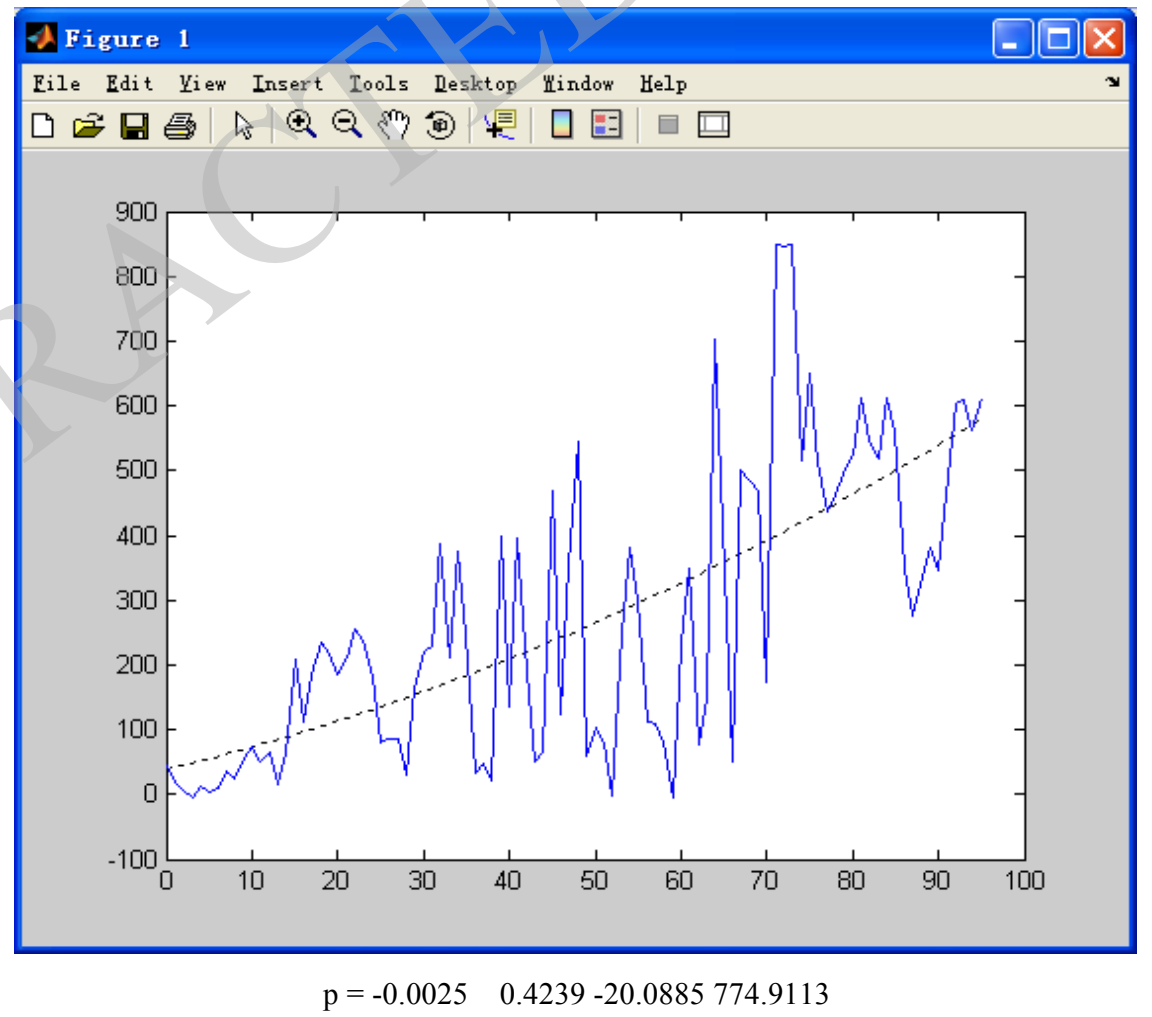

Fig. (15). Contd... 
PA5

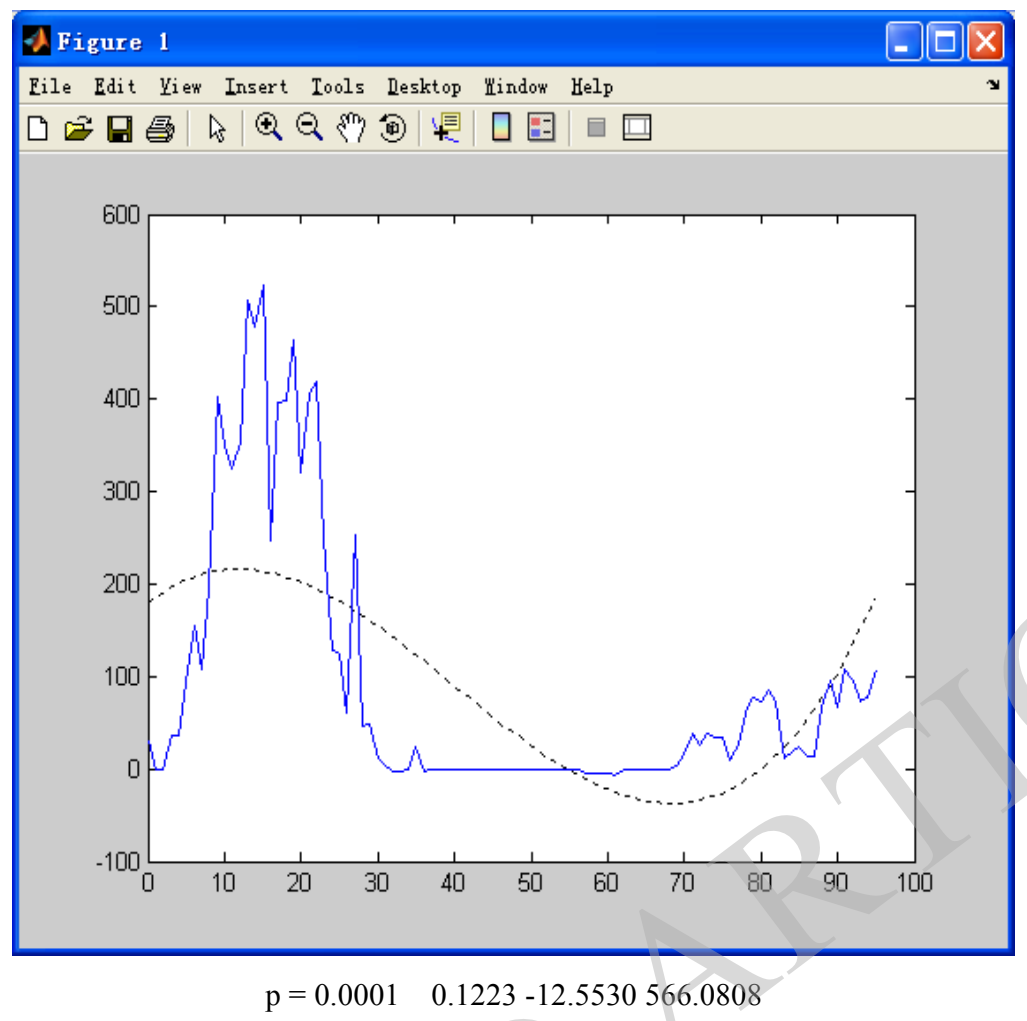

PB

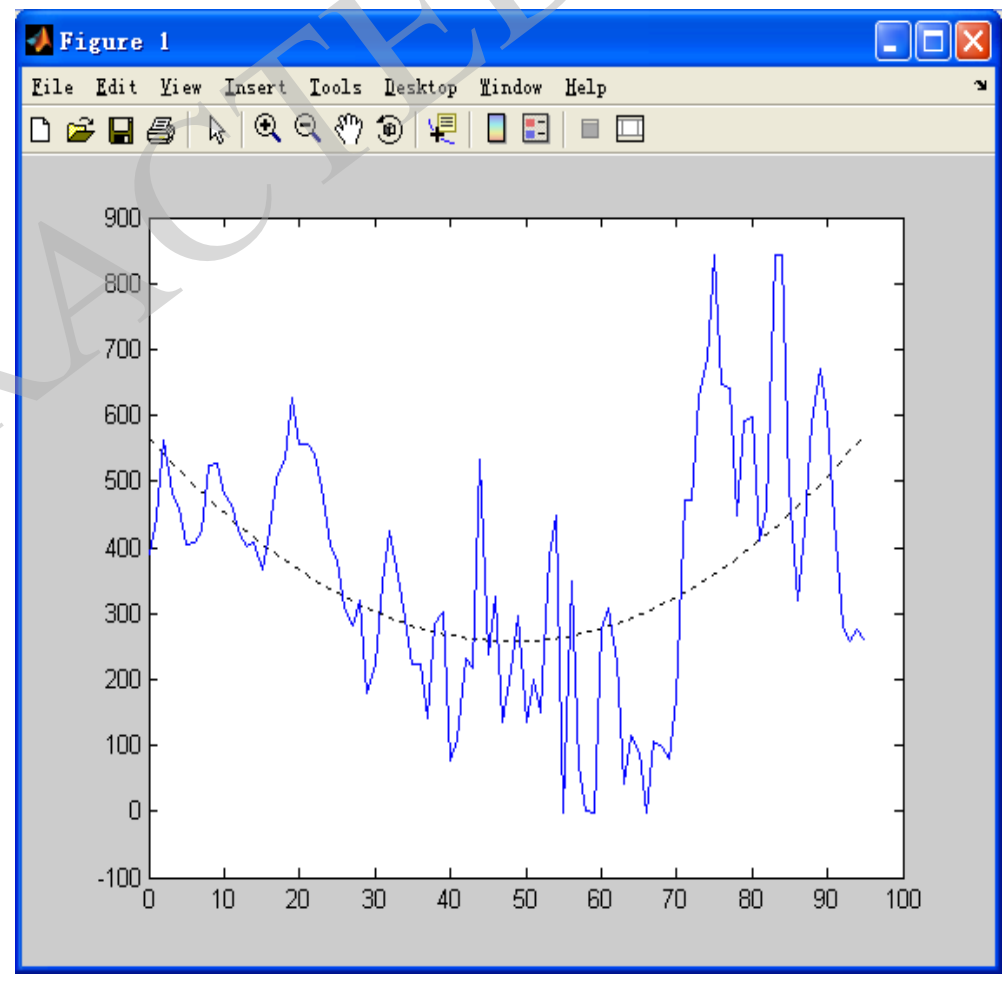

$\mathrm{p}=0.0028 \quad-0.3375 \quad 6.7780178 .4517$

Fig. (15). Contd... 
PC

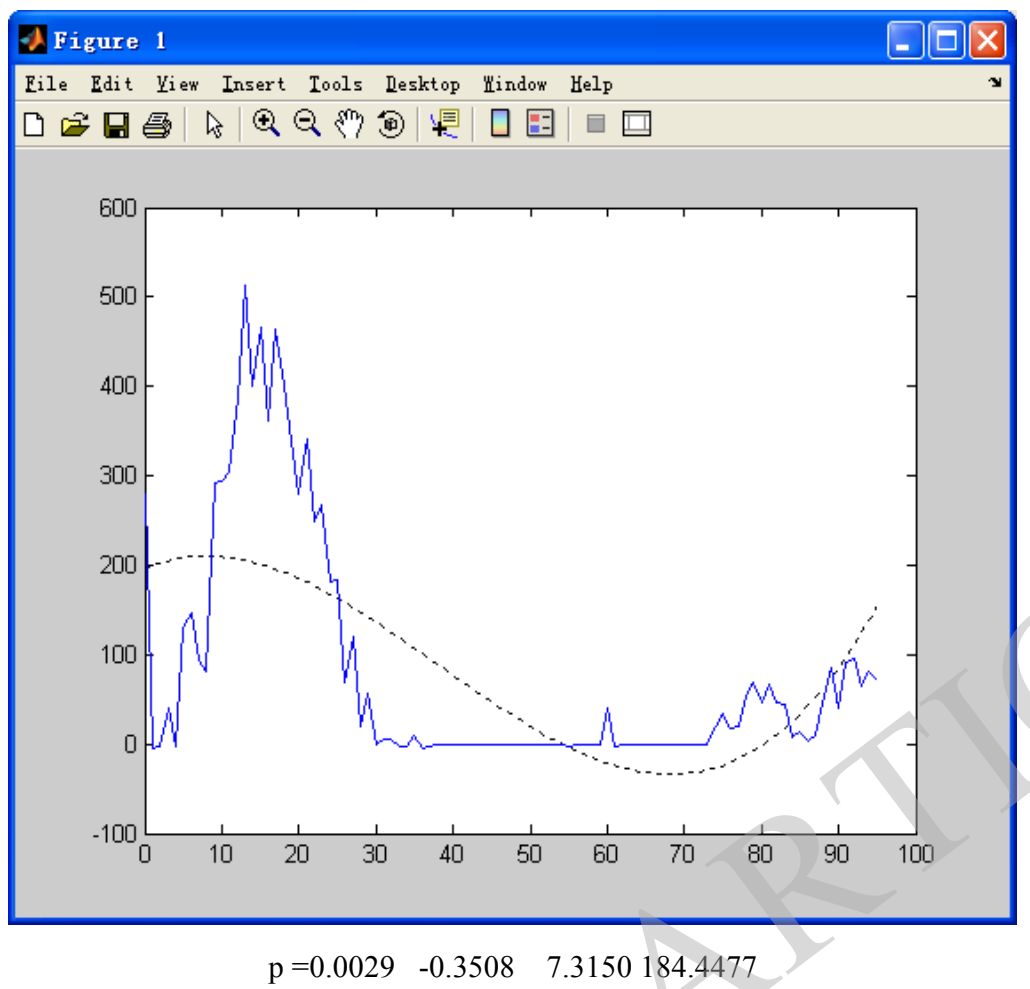

PD

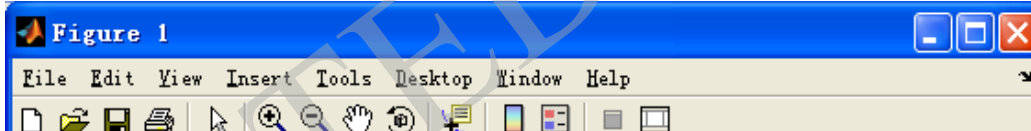

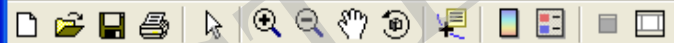

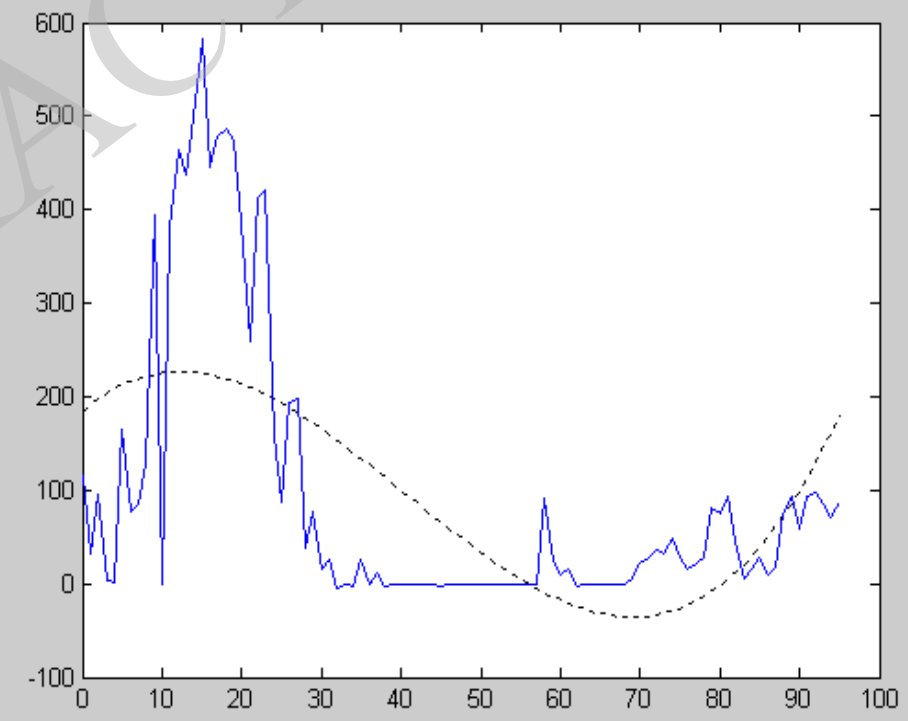

$p=0.0022 \quad-0.2556 \quad 3.6482196 .3227$

Fig. (15). Contd... 


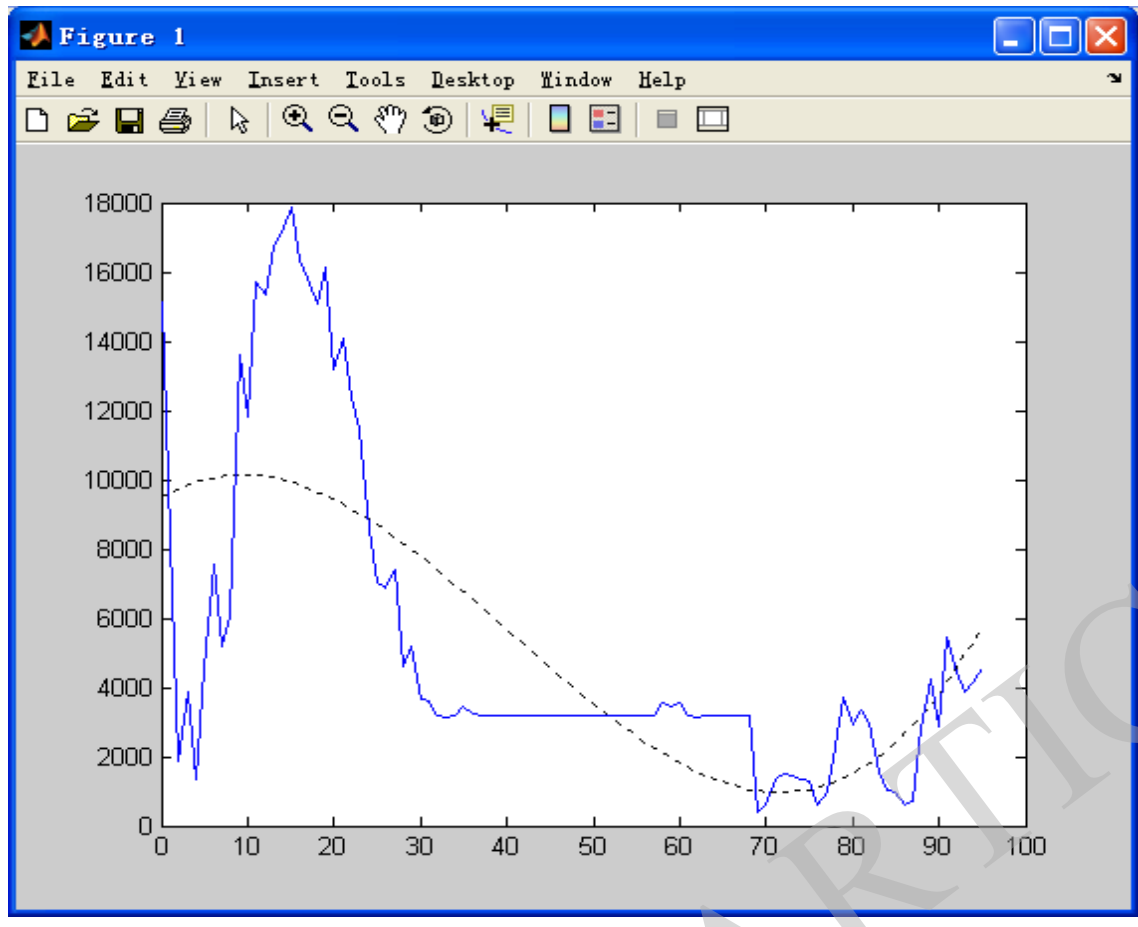

$\mathrm{P}=1.0 \mathrm{e}+003 *(0.0001-0.00920 .1505$ to 9.4748$)$

Fig. (15). Fitting Curve and Fitting CoefficientP A fitting curve: $y=k_{1} t^{3}+k_{2} t^{2}+k_{3} t+k_{4}$.

Table 3. Fitting Coefficients.

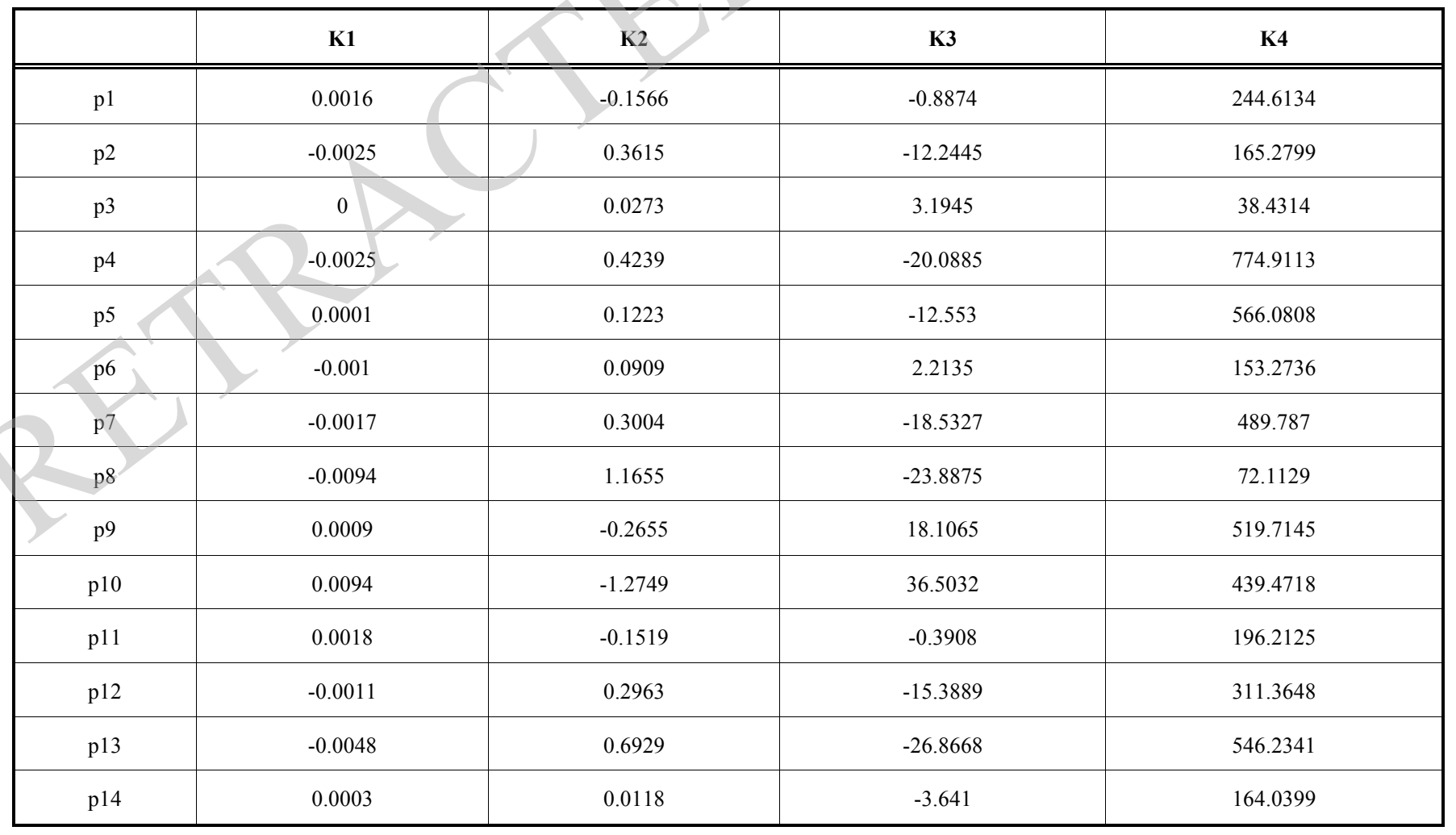


Table 3. contd...

\begin{tabular}{|c|c|c|c|c|}
\hline p15 & 0.0043 & -0.6921 & 32.5371 & -44.3881 \\
\hline p17 & 0.0034 & -0.5581 & 21.592 & 158.242 \\
\hline p19 & 0.0021 & -0.1869 & 4.2237 & -18.7512 \\
\hline $\mathrm{p} 20$ & -0.0004 & 0.2071 & -16.3004 & 461.2765 \\
\hline p21 & -0.0011 & 0.1485 & -7.1552 & 541.80 \\
\hline $\mathrm{p} 25$ & 0.0015 & -0.0728 & -1.1318 & 106.3212 \\
\hline p26 & -0.0008 & 0.2624 & -21.9567 & 627.6076 \\
\hline p27 & 0.001 & -0.0469 & -0.7941 & 36.9581 \\
\hline p28 & -0.0107 & 1.3132 & -36.5566 & 621.5351 \\
\hline
\end{tabular}

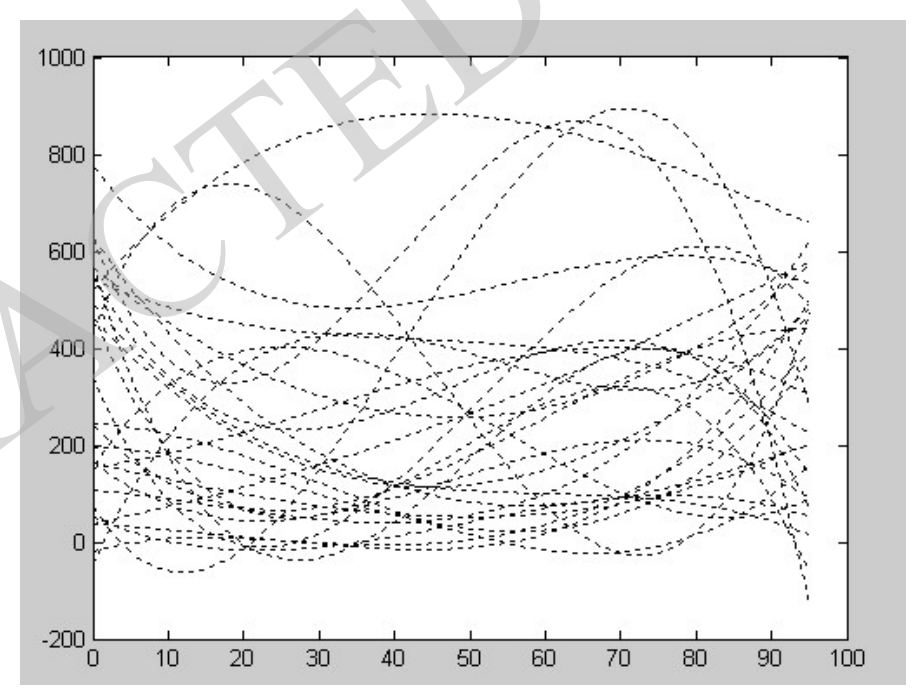

Fig. (16). 28 Fitting Curves of PA.

\subsection{Error Analysis}

Taking PA 2006-5-23 as an example, the comparison between the forecast values and the actual values: $t=0$, $\mathrm{y}=210.2515$; the actual value of $208.2188 ; \mathrm{t}=1$, $y=201.7483$; the actual value of $229.125 ; \mathrm{t}=2, \mathrm{y}=193.573$; the actual value of $229.125 ; \mathrm{t}=3, \mathrm{y}=185.7205$; the actual value is 187.875 . Error $=$ (predictive value - the actual value) / actual value, the error obtained is less than $15 \%$, compliance with the requirements of the title for the model error.

\section{COMPARED ADVANTAGES OF THREE KINDS OF MODELS}

(1) ARMA neural network model is a short-term prediction model with a relatively high degree of precision; it is suitable for various types of time series. And it is a flexible prediction model; the applicability of statistical methods can be used for a series of model test in the process of modeling, to constantly adjust the numbers order of the model, till to achieve satisfactory results. ARMA model is used as the actual forecasting and can update prediction, 
observation and forthcoming values join the model in time, the model establishes again and makes the new predictions again, which play an important role in forecasting. Especially for the wind power forecasting, time series analysis can better capture the variation law of wind power and the ideal forecasting effect can be obtained. But this model is restricted, ARMA model identification is often needed more than 50 months or yearly history data, so the data collection needs to spend some time.

(2) The neural network model has the characteristics of distributed storage and parallel processing, fault tolerance and so on, which is very effective for solving complex problems and is ideal in the prediction precision for shortterm wind power prediction. But because BP neural network training results are abnormal sensitivity to the initial weight matrixes and threshold vectors, using MATLAB toolbox initialization the neural network, the initial weight matrixes and the threshold vectors are randomly generated, resulting in each run predictions are not the same and in serious impact on prediction research, and possibly in the network weights and threshold parameters optimization into the local minimum, the network generalization ability and adaptation ability is poor, even bring a halt and be unable to meet the accuracy requirements.

(3) The wavelet analysis can easily determine the characteristic of frequency domain and time domain location of the wavelet family. Because the wavelet transform is a time-frequency analysis, so it can also describe the signal characteristics of time and frequency. With the rapid development of computer technology, wavelet analysis has more and more obvious advantages with its good performance analysis and accurate analysis methods in the wind power forecast. However, wavelet analysis has certain requirements for analysis amount.

\section{CONCLUSION}

Through the analysis and the comparison of the three methods we recommend using the wavelet analysis to determine the real-time prediction model, and we recommend using neural network model for the recently predictions. In order to construct the real-time prediction of higher prediction accuracy, we recommend combining the wavelet neural with the network model. This model decomposes the original waveform by wavelet function in the different scales; the periodic components decomposed are predicted with the time series, the rest uses the neural network prediction, the signal sequences are reconstructed to obtain the predicted results of the complete wind power. Joined the differential evolution algorithm in neural network learning process, improve the speed of convergence and solve the local minimum problem, through the analysis and modeling it have proved that the combination algorithm can be targeted to the accurate prediction of wind power.

\section{CONFLICT OF INTEREST}

The authors confirm that this article content has no conflict of interest.

\section{ACKNOWLEDGEMENTS}

\author{
Declared none.
}

\section{REFERENCES}

[1] Yao Xingjia.Wind Power Testing Technology, Beijing: Publishing House of Electronics Industry, 2011.

[2] Chen Guochu, Wang Peng, Xu Yufa, Yu Jinshou. Short Term Wind Power Prediction Model Based On Hybrid Wavelet Decomposition, Fourteenth Volume Third Issue.

[3] Peng Yuhua, Wavelet Transform and Engineering Application, Beijing: Science Press, 1999;

[4] Wang, Yuanzhi, Zhang, Hui; Jiang, Shanhe; Sun, Lijuan.Fuzzy support vector machine based on improved possibilistic c-means clustering algorithm in kernel space [J]. Journal of Information and Computational Science, v 7, n 2, p 585-591, February 2010.

[5] Yang Guixing, Chang Xiqiang, Wang Weiqing, Yao Xiuping.On The Tccuracy of Wind Power Forecasting Discuss Prediction System, Power Grid And Clean Energy Twenty-seventh Volume First Issue.

[6] Fan Gaofeng, Wang Weisheng, Liu Chun, Dai Huizhu.The Wind Power Forecast Based On Artificial Neural Network, Chinese School Of Electrical Engineering, Twenty-eighth Volume Thirtyfourth Issue. 